\title{
Cavernous Carotid Artery Pseudoaneurysm Following a Radical Cavernous Sinus Resection
}

\author{
Miki Katzir $^{1}$ Ziv Gil ${ }^{2}$ José Enrique Cohen ${ }^{3}$ Gill Efraim Sviri ${ }^{1}$ \\ ${ }^{1}$ Department of Neurosurgery, The Technion, Israel Institute of \\ Technology, Haifa, Israel \\ 2 Department of Otolaryngology Head and Neck Surgery, The \\ Technion, Israel Institute of Technology, Haifa, Israel

\begin{abstract}
Address for correspondence Gill E. Sviri, MD, MSc, Department of Institute of Technology, Haaliya Hashniya 8, Haifa, Israel, 3109601 (e-mail: g_sviri@rambam.health.gov.il).
\end{abstract} \\ Neurosurgery, Rambam Health Care Campus, The Technion, Israel
}

${ }^{3}$ Department of Neurosurgery, Hadassah-Hebrew University Medical Center, Jerusalem, Israel

J Neurol Surg Rep 2016;77:e102-e105.
Abstract
Keywords
- pseudoaneurysm
- cavernous sinus
- endovascular
- stent
- aneurysm
- rhabdomyosarcoma

latrogenic cavernous carotid pseudoaneurysms are a special group among other intracranial aneurysms. They can occur during the dissection phase of the surgery if the tumor encases a vessel. Complications of their rupture as hemorrhage or stroke are life threatening. Early recognition and treatment is mandatory to avoid catastrophic sequelae. We present the successful diagnosis and endovascular treatment of a postoperative cavernous carotid pseudoaneurysm following radical cavernous sinus resection.

\section{Introduction}

Cavernous carotid artery (CCA) aneurysms are considered a distinct group from all other intracranial aneurysms as the completeness of the cavernous sinus (CS) walls is responsible for their low subarachnoid hemorrhage $(\mathrm{SAH})$ risk. $^{1}$

Their etiology can be idiopathic, iatrogenic, traumatic, and rarely infectious. ${ }^{2}$

In 1924, Barth described the classic triad of signs for traumatic cavernous carotid pseudoaneurysms (CCPA); it includes severe epistaxis often years following the injury delaying diagnosis by blurring the link between them, monocular vision loss, and skull base fracture. ${ }^{3}$ Direct trauma to the wall of the CCA can lead to the development of a carotid cavernous fistula (CCF), however; additionally injuring the wall of the CS will lead to pseudoaneurysm formation by the fibrous organization of the locally contained hematoma within the remains of the CS. ${ }^{4,5}$ Knowledge of skull base surgical anatomy hand in hand with superb surgical dexterity grants us the safety in drilling, a cornerstone during the exposure part of a skull base surgery. Nevertheless, vascular laceration can occur; more commonly during the dissection phase if the tumor encases a vessel. We describe the incidental discovery of a CCPA during a routine contrastenhanced computed tomography (CT) imaging done as part of the fever workup on an 11-year-old patient, 21 days following craniotomy for a skull base rhabdomyosarcoma tumor. We emphasize the importance of active search for these aneurysms following surgery as aneurysmal formation can occur even in the absence of obvious intraoperative vessel injury hemorrhage or clinical symptoms.

\section{Case Report}

An 11-year-old child diagnosed with a rhabdomyosarcoma in his left infratemporal fossa and parapharyngeal space underwent tumor resection that included neoadjuvant chemotherapy, combined transcervical-infratemporal fossa approach, and complete resection of tumor followed by radiotherapy and chemotherapy. A follow-up magnetic resonance imaging scan a year later disclosed a new lesion involving the lateral part of the left CS and foramen ovale (FO) necessitating reoperation (-Fig. 1A-F).
License terms

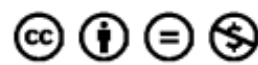

received
December 23, 2015 accepted after revision April 18, 2016

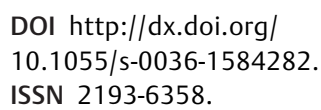

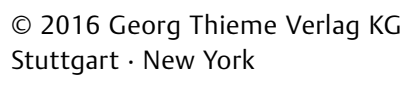




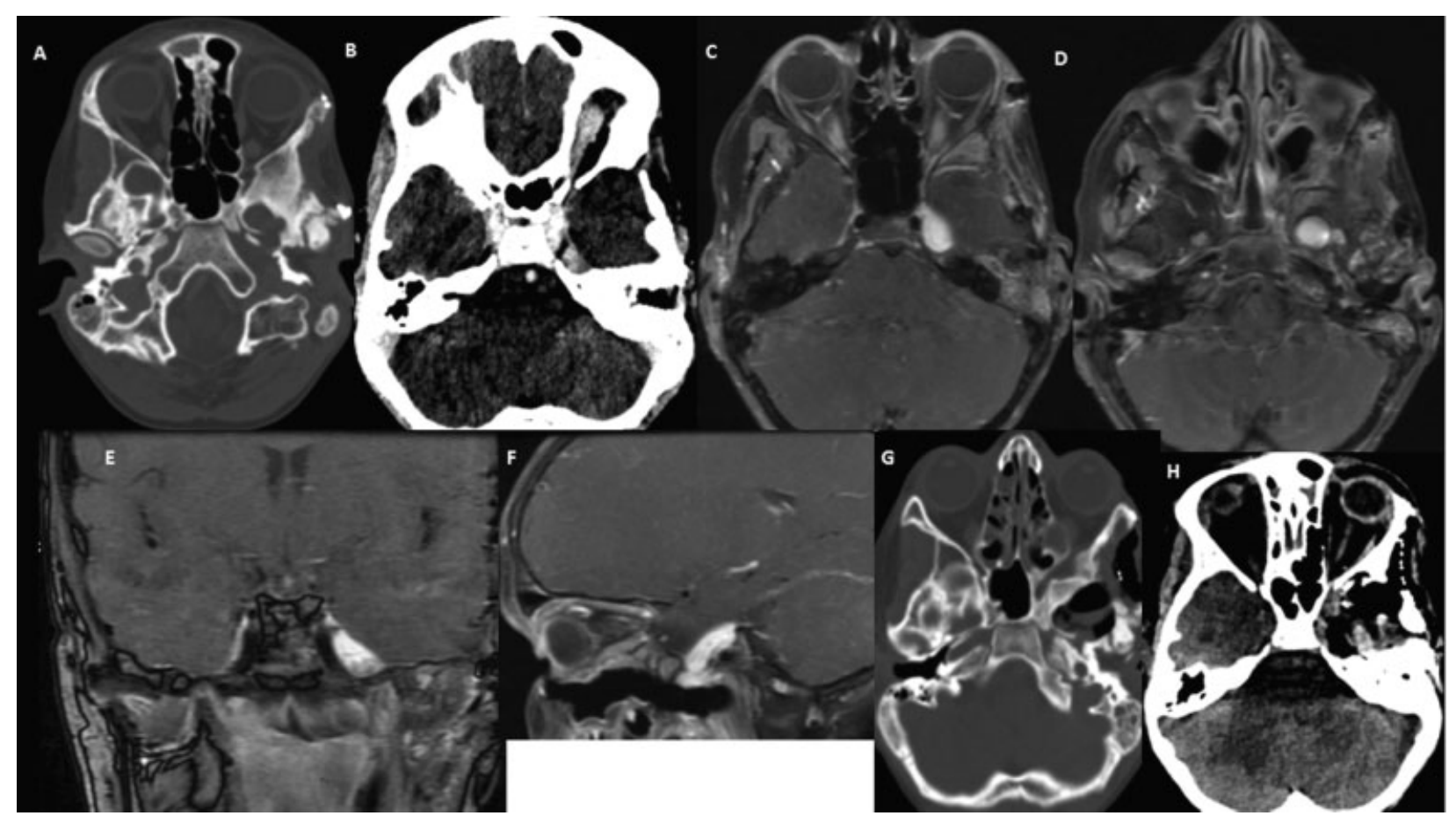

Fig. 1 (A-F) Preoperative and ( $G$ and $H$ ) postoperative $C T$ and MRI showing the tumor. (A: Axial CT scan showing enlarged left foramen ovale. B: Contrast-enhanced axial CT and MRI [C and D]: Scans showing left CS mass. [E and F]: Coronal and sagittal contrast enhanced MRI scans. [G and H]: Axial CT scans showing no bleeding or brain infarcts). CT, computed tomography; CS, cavernous sinus; MRI, magnetic resonance imaging.

A left frontotemporal craniotomy combined with zygomatic osteotomy was followed with ligation of the middle meningeal artery at the foramen spinosum via a subtemporal extradural approach. The anterolateral wall of the FO was drilled out to allow mobilization of the tumor that invaded the mandibular division of the trigeminal nerve (V3). Proximal vascular control was achieved via unroofing the petrous internal carotid artery (ICA) near the junction of the Glasscock and Kawase triangles. The greater and lesser petrosal nerves were sectioned to prevent facial nerve injury and to improve petrous carotid exposure. Elevating the external leaf of the CS posterolaterally exposed the tumor and V3 within Meckel cave. The lateral bony wall of the superior orbital fissure was drilled out starting from the foramen rotundum (FR) to its upper free edge. To achieve mobilization of trigeminal's nerve maxillary branch at FR its anterolateral aspect was drilled out.

The three branches of the trigeminal nerve were incised at their foramina's entry point and the trigeminal nerve at its entry point to Meckel cave.

An intradural approach to the CS was used to allow medial mobilization and dissection of the tumor. The dura was split just below the dural entry point of the oculomotor nerve, allowing identification of the CCA; the tumor was separated from the medial CS and CCA from proximal to distal using blunt and sharp dissection allowing a complete mobilization and resection of the tumor from the CCA with preservation of the middle layers of the CS, oculomotor, and trochlear nerves.

However, due to tumor invasion, the abducens nerve has been incised adjacent and lateral to the CCA. During the dissection of the tumor from the medial part of the CS a minor arterial and venous bleeding were encountered and controlled with application of Surgicel (Ethicon, Somerville, New Jersey, United States) and gentle pressure. Nevertheless, we could not identify direct injury to the wall of the CCA.

After complete tumor resection, Meckel cave entry point was sealed with muscle piece and Tachosil patch (Takeda Austria GmbH, Linz, Austria), dural closure was achieved using 4/0 Surgilon suture (Medtronic, Dublin, Ireland) and a vascularized temporalis muscle and pericranial flap were mobilized to the middle cranial fossa.

The patient recovered well and a postoperative CT scan disclosed minor fluid collection in the temporal fossa, but no bleeding or brain infarcts (-Fig. 1G-H). Three weeks following the surgery the patient presented with fever and as a part of the fever workup a contrast-enhanced CT scan was done, which disclosed a mass at the left CS (- Fig. 2A). A CT angiography (CTA) ( - Fig. 2B-D) followed by digital subtraction angiography (DSA) ( - Fig. 2E) revealed a left CCPA measuring $18 \mathrm{~mm} \times 3.5 \mathrm{~mm}$. The pseudoaneurysm was treated endovascularly using a single-covered stent allowing preservation of the lumen with no further complication (-Fig. 2F). An MRI done 3 months postoperatively shows tumor resection ( - Fig. 3A-C).

\section{Discussion}

Saccular traumatic pseudoaneurysms occur due to arterial wall laceration, contained by an organized extraluminal hematoma thus lacking a true wall, a fact that predispose them to rupture more easily than idiopathic or traumatic fusiform aneurysms in which a true wall exists, but 

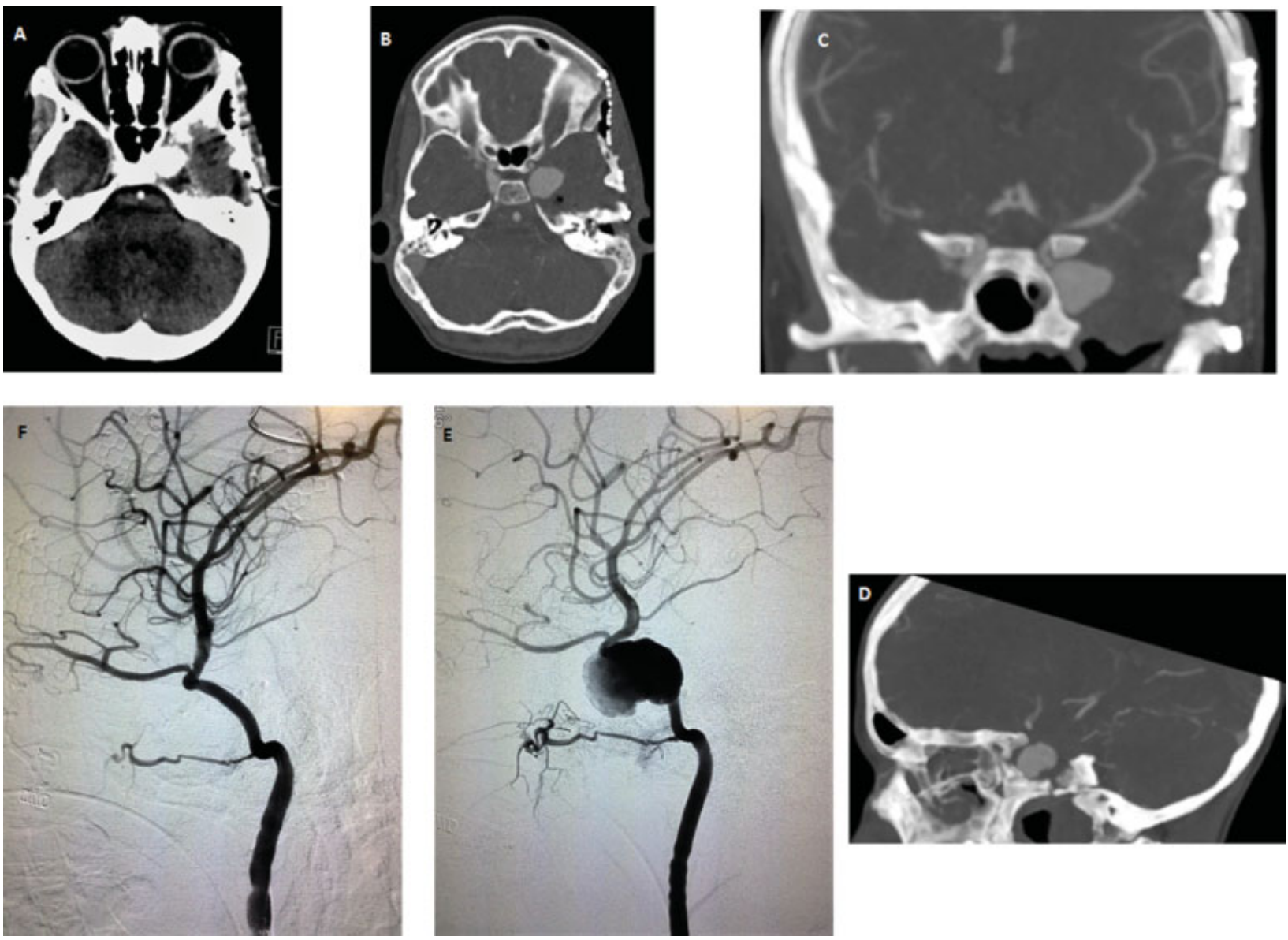

Fig. 2 (A) Contrast-enhanced axial CT show left CS mass. (B-D) Axial, coronal, and sagittal CTA scan. (E and F) Left carotid digital subtraction angiography before and after stenting of the pseudoaneurysm. CT, computed tomography; CTA, computed tomography angiography; CS, cavernous sinus.

adventitial thinning caused by peeling the tumor off the adventitia causes vascular dilatation; their rupture risk is lower than saccular aneurysms. ${ }^{6}$

Hemorrhagic or embolic complications ${ }^{7-10}$ are common for these rare pseudoaneurysms and they face the patient at great risk if being unrecognized, thus, the immediate recognition of the above symptoms and treatment is prerequisite to prevent fatal complications.
Iatrogenic CCPA is mostly described in relation to transsphenoidal procedures due to the proximal relation between the CCA, the wall of the sphenoid sinus and the sella turcica. ${ }^{9,11,12}$ It is rarer following skull base surgery due to the relative paucity of the procedure, nevertheless, its life-threatening consequences such as rupture, severe hemorrhage, pituitary apoplexy, stroke, and death necessitate urgent diagnosis and treatment even for an incidentaloma, such as our case.
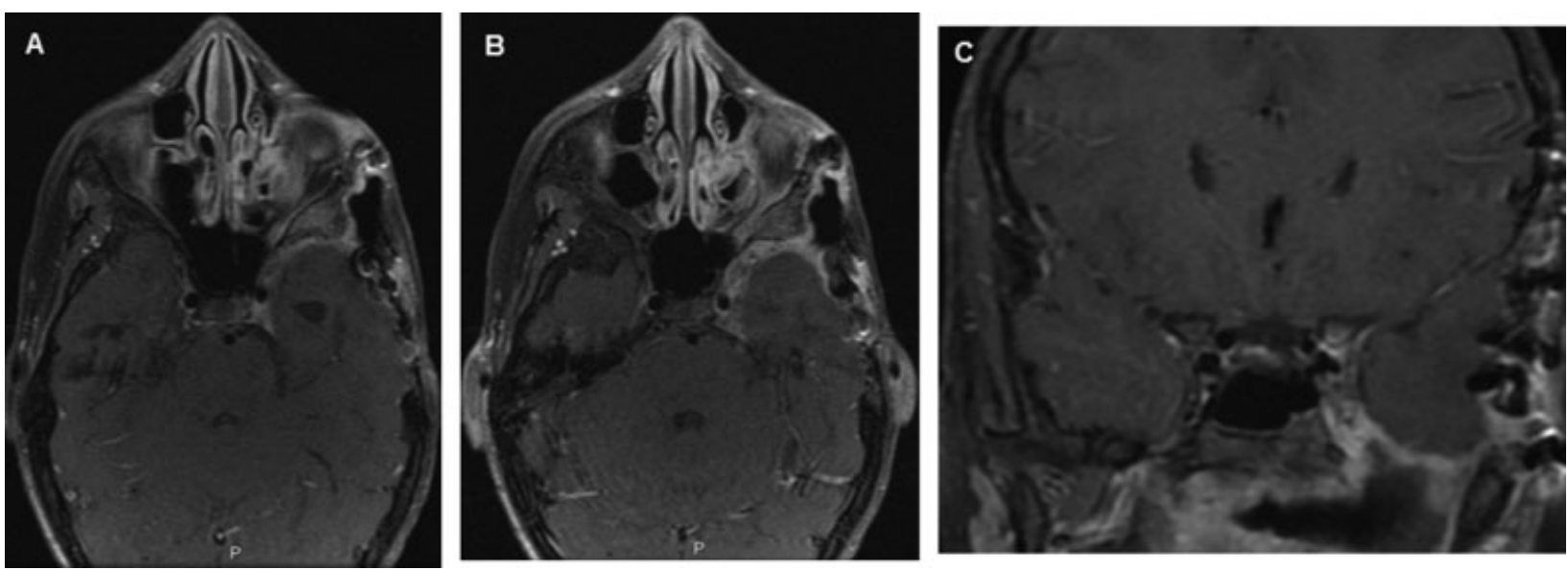

Fig. 3 (A-C) Postoperative axial and coronal contrast enhanced MRI scans. MRI, magnetic resonance imaging. 
One report exists, where a patient refusal to treat his pseudoaneurysm resulted in his death from severe epistaxis 2 years following surgery, thus making the always optional conservative management treatment irrelevant. ${ }^{9}$ When suspicion of vascular injury arises during surgery, it is logical to perform a postoperative vascular imaging, such as CTA or DSA. ${ }^{5,13,14}$ In our case, in the absence of major intraoperative bleeding or direct injury to the arterial wall, such suspicion did not arise.

Despite the uneventful operative and postoperative course, a pseudoaneurysm measuring $18 \mathrm{~mm} \times 3.5 \mathrm{~mm}$ was discovered and later stented with a single stent.

Knowing that postsurgical changes can blur minute vascular abnormalities and that that the natural history of pseudoaneurysms is to grow over time we recommend to perform a delayed vascular imaging, specifically in search for this feared complication even in the absence of suggestive symptomatology and a negative immediate postoperative imaging.

In the absence of intact CS walls after surgical resection, the aneurysm will not be contained within the CS wall and might rupture directly into the epidural space leading to the formation of acute epidural hematoma and subsequently brain herniation and death within a very short period of time.

\section{Conclusion}

Rapid formation of CCPA can occur following CS surgery, even with uneventful surgery and without obvious injury to the CA wall. The formation is asymptomatic and can end in a catastrophic bleeding as the aneurysm is not contained in the CS walls. Therefore, postoperative imaging directed to identify aneurysmal formation should be conducted for early diagnosis and treatment.

\section{References}

1 Kupersmith MJ, Hurst R, Berenstein A, Choi IS, Jafar J, Ransohoff J. The benign course of cavernous carotid artery aneurysms. J Neurosurg 1992;77(5):690-693
2 Glaiberman CB, Towbin RB, Boal DK. Giant mycotic aneurysm of the internal carotid artery in a child: endovascular treatment. Pediatr Radiol 2003;33(3):211-215

3 Barth G. Todliche spatblutung aus der carotis interna nach schadeltrauma. Dtsch Med Wochenschr 1924;50:875-876

4 Bavinzski G, Killer M, Knosp E, Ferraz-Leite H, Gruber A, Richling B. False aneurysms of the intracavernous carotid artery-report of 7 cases. Acta Neurochir (Wien) 1997;139(1):37-43

5 Ciceri EF, Regna-Gladin C, Erbetta A, et al. Iatrogenic intracranial pseudoaneurysms: neuroradiological and therapeutical considerations, including endovascular options. Neurol Sci 2006;27(5): 317-322

6 Sutton LN. Vascular complications of surgery for craniopharyngioma and hypothalamic glioma. Pediatr Neurosurg 1994;21 (Suppl 1):124-128

7 Buerke B, Tombach B, Stoll W, Heindel W, Niederstadt T. Magnetic resonance angiography follow-up examinations to detect iatrogenic pseudoaneurysms following otorhinolaryngological surgery. J Laryngol Otol 2007;121(7):698-701

8 Pozzati E, Giuliani G, Poppi M, Faenza A. Blunt traumatic carotid dissection with delayed symptoms. Stroke 1989;20(3): 412-416

9 Raymond J, Hardy J, Czepko R, Roy D. Arterial injuries in transsphenoidal surgery for pituitary adenoma; the role of angiography and endovascular treatment. AJNR Am J Neuroradiol 1997;18(4): 655-665

10 Dellen JR. Traumatic carotid aneurysms of the cavernous sinus. In: Dolenc VV, ed. The Cavernous Sinus. A multidisciplinary approach to vascular and tumorous lesions. Wien New York: Springer; 1987: 233-240

11 Ahuja A, Guterman LR, Hopkins LN. Carotid cavernous fistula and false aneurysm of the cavernous carotid artery: complications of transsphenoidal surgery. Neurosurgery 1992;31(4):774-778, discussion 778-779

12 Cappabianca P, Briganti F, Cavallo LM, de Divitiis E. Pseudoaneurysm of the intracavernous carotid artery following endoscopic endonasal transsphenoidal surgery, treated by endovascular approach. Acta Neurochir (Wien) 2001;143(1): 95-96

13 Ciric I, Ragin A, Baumgartner C, Pierce D. Complications of transsphenoidal surgery: results of a national survey, review of the literature, and personal experience. Neurosurgery 1997;40(2): 225-236, discussion 236-237

14 Laws ER Jr. Vascular complications of transsphenoidal surgery. Pituitary 1999;2(2):163-170 